\title{
The safety assessment of lifting equipment using artificial intelligence methods
}

\author{
$A$ Lyapin $^{1, *}$ \\ ${ }^{1}$ Don State Technical University, 1, Gagarin square, 344000, Rostov-on-Don, Russia
}

\begin{abstract}
The article is devoted to the problem of using artificial neural networks to assess the risk of developing emergencies during the operation of lifting crane equipment. The data sources are telemetric measurements from microcontroller load limiters, as well as data from technical and daily inspections of equipment condition, in the last case the data may be fuzzy.
\end{abstract}

\section{Introduction}

The purpose of analysis of the telemetric data received on equipment and similar devices of a lifting crane, as well as indicators of devices from the local security system of the facility, is to develop and implement the technology for assessing any risk of emergencies at the current moment and predicting the risk for the near future. Given a weak formalism of the input information (in terms of fuzzy sets), as well as a dynamic discrete nature of information flow from safety equipment, and based on the examples of the best known practices, the best performance can be achieved with the application of machine learning techniques, neural networks, processing and clustering big data combined with fuzzy data analysis methods for decision making. These approaches had been developed in [1-3].

The main deliverables of the proposed approach are:

- the study of application for neural network data analysis in the framework of fuzzy input information for corresponding classification according to the severity of the accident consequences and the rate of a hazardous event probability;

- the investigation of mechanisms for statistical data collecting, processing of telemetry data for a number of parameters, including the problems of recognition and classification of historically accumulated data by the types and changes nature in telemetry parameters;

- the construction and study of the general problem for assessing emergencies risk development at hazardous facilities using a multi-layer neural network of direct propagation, with the solution of 2 previous problems as inputs.

Note that the first research problem has a solution based on fuzzy inference, including the stages of fuzzification, application of fuzzy production rules and defuzzification, for example in accordance with the Takagi-Sugeno method. The most developed approaches here are the use of the 5-layer Takagi-Sugeno-Kanga (TSK) neural network and the three-layer WangMendel network $[4,5]$. Network training can be carried out both with a teacher and without a teacher based on the clustering of input data.

\footnotetext{
* Corresponding author: lyapin.rnd@yandex.ru
} 
To solve the second problem, the most applicable to the problems of classification and clustering the classical multilayer neural networks and recurrent neural network can be used. The second option is preferable for analyzing time series and signals [6].

In general, the technology of neural networks is the most widely used in the transport systems risk assessment problems [7-8], construction [9-10], evaluation of material properties [11], information technologies [12] and others.

\section{Data and methods}

The process of artificial neural networks studying for solving the general problem on predicting the risk of emergencies for lifting crane devices has the following stages of implementation:

- Structuring and analysis of the input information;

- Assessment of mechanisms for transforming the input information into target indicators for solving the problem of predicting the risk of emergencies;

- Determining the type and structure of the artificial neural network (ANN) elements;

- Learning neural network on the reference data set;

- Neural network performance check.

The input information of neural network is divided into the following types:

a) Information from questionnaires

Features of the information flow:

- Lack of historically accumulated data;

- Fuzzy nature of information in the form of linguistic variable values ;

- Availability of an expert mechanism for assessing the influence of input information on the output parameters of the network, including a system of production rules of fuzzytype and coefficient-type additive models for obtaining the final result;

b) Information of a monitoring nature, accumulated during the entire operation of the crane safety device, containing sections of general information, such as "Statistics of operating cycles", "Operating time", "Quality of control", etc., as well as operational information recorded frames with an interval of $1 \mathrm{~s}$, some of which are used in the neural network model:

- time indication;

- boom length indication;

- indication of the lifted load;

- indicator of rated lifting capacity for the current value of boom length and reach;

- indication of the platform rotation angle;

- $\quad$ sign of the presence of power lines;

- information about the coordinate protection parameters set;

- warning signals indicating that the load exceeds the level of $90 \%$ of the nominal allowable value;

- $\quad$ entry of the load level into the range from 100 to $110 \%$

- warning signals indicating the approach to the permissible boundaries of coordinate protection;

- $\quad$ signal of device operation for complete stop of mechanisms.

Features of the information flow:

- Dynamic structure of information;

- The presence of a random nature due to external influences (wind load, random vibrations of the lifting mechanisms), possible accidental influences on the crane 
operator's control;

- Availability of historical data, most of which is related to accident-free operating modes of the crane and almost missing data in the case of an emergency;

- Ability to supplement the monitoring data for the selected characteristic time interval in order to retrain the neural network.

Thus, from the analysis of the input information, it was decided to use ANN for the analysis of information flows of both types and obtaining results with an assessment of the risk of an emergency when using a crane. However, the structural elements of such network should be different for various information flows.

\section{Results and discussions}

To solve the problem of the lifting crane compliance with industrial safety requirements at levels [2] (does not match - red; not fully, take measures to reduce the risk - yellow; matches - green) there built a 4-layer neural network that solves the problem of multi-parameter classification based on input information from questionnaires.

Used: programming language - Python, interpreter version 3.6; Tensorflow version 1.8.0 is a low-level machine learning framework that includes Keras of version 2.1.6 - a deep learning library, as well as some additional packages of mathematical and infrastructure nature.

Variants of answers by categories were used as network inputs:

- Life cycle of equipment?

- Timing of industrial safety expertise of the crane?

- Availability of insurance?

- Timing and results of the last maintenance?

- Assessment of the level of flange wear in comparison with its original thickness?

- Are there cracks of any size on drums and hooks, welds and base metal?

- Level of wear of a drum? etc.

the answers to which are the values of the corresponding linguistic variables. The total number of inputs is 100 . The value of the linguistic variable marked in the checklist is given by the level - one, the rest - by zeros.

The general structure of the neural network by layers is shown in Figure 1. The number of fully connected layers is 4 . One layer of the Dropout class to prevent overfitting of the neural network is a drop layer with a coefficient of 0.1 . The activation function on the inner layers is a hyperbolic tangent, in the output layer - a threshold one. Number of outputs - 6 corresponding to the binary coding of 3 levels of severity of accident consequences and 3 levels of probability of a hazardous event. To assess the performance of the model, the function MSE was used - the root-mean-square error between the output and reference values of neurons. The method of stochastic gradient descent is used as an optimizer. The presented structure is optimal as a result of a number of studies on ANN parameters:

- activation functions on layers;

- initializers of scales;

- loss functions;

- optimizers;

- number of neurons on hidden layers.

The network was trained using the error backpropagation method. Note that the proposed procedure is rather cumbersome due to the impossibility of training the system with a complete set of input reference values. For training, we selected the elements located near the hyperplanes section according to the categories of the severity levels of the accident 
consequences and the levels of the probability of a hazardous event. Checking the remaining options of input information was performed at the level of neural network trained validation and demonstrated the effectiveness of the proposed approach.

To implement the analysis of statistical data accumulated during the entire operation of the crane safety device, 2 approaches are considered. The first uses a recurrent neural network (RNN).

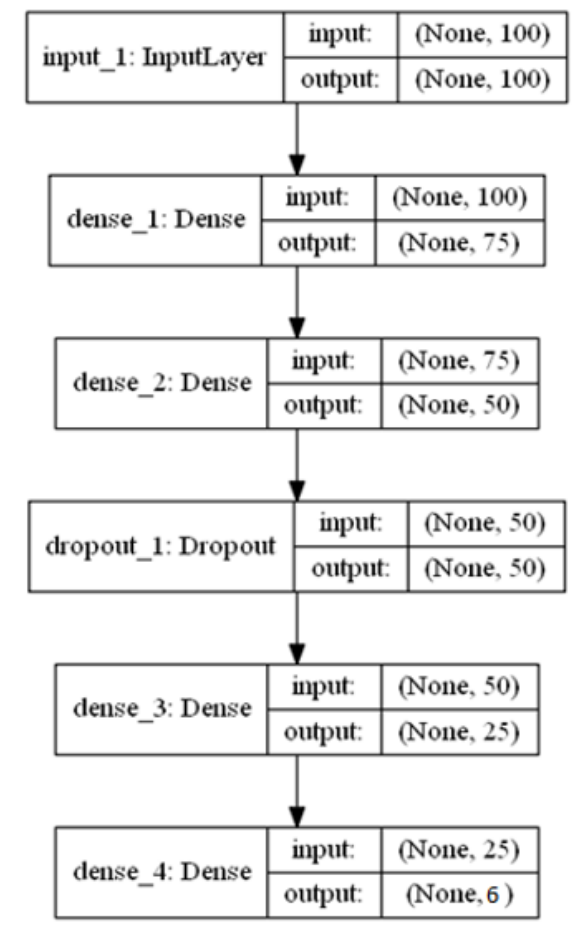

Fig. 1. General diagram of a neural network.

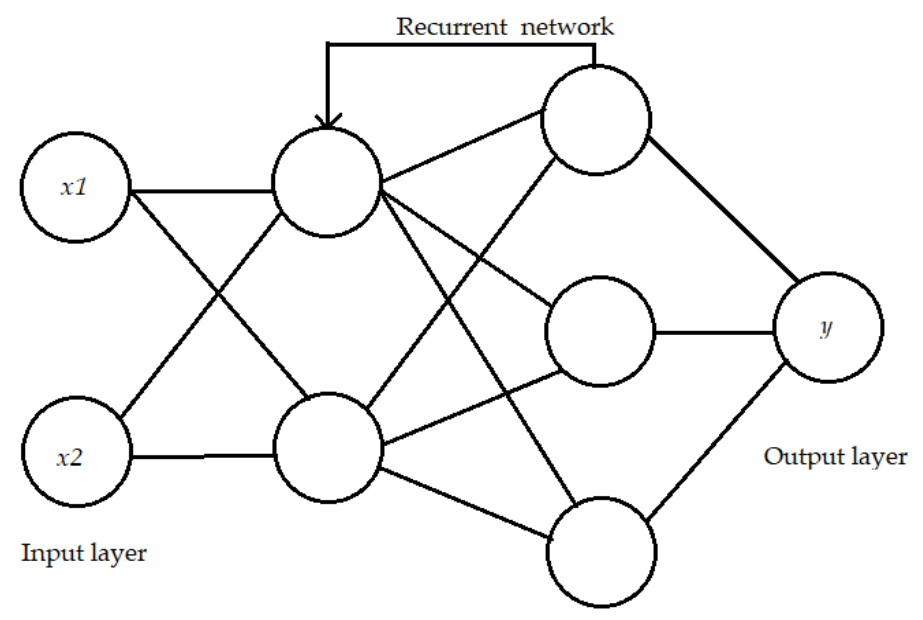

Hidden layers

Fig. 2. Scheme of a recurrent neural network. 
The network inputs (Figure 2) are the values of the registered parameters by the safety device, listed above, within the time window of the specified width $\Delta \mathrm{T}$. The optimal number of points of the time base of the signal within the selected window was determined from 20 to 30. The number of neurons in the output layer was selected from 6 to 10 in accordance with the number of degrees of freedom of the crane mechanism (lifting, movement of the carriage, turn, movement on the rails) and the number of restrictions in the process of moving cargo (carrying capacity, coordinate protection, the presence of power lines). The main task is to classify signals based on unsupervised learning, revealing their internal organization. The number of neurons in the two hidden layers was proportionally reduced to create a trapezoidal topological network structure. The classification of recorded signals made it possible to distinguish classes according to the types of movement of crane mechanisms, as well as a simultaneous change in the recorded characteristics.

A scheme for further classification within the limits of each recorded signal is proposed. For this, the construction of separate classifier networks was carried out. The already used time base of the selected signal (from 20 to 30 samples) is fed to the input of the network, on the basis of which the subclasses are determined: uniform change of parameters, change with acceleration and random change.

Thus, the final RNN outputs are signs of belonging to the type of telemetry parameters change (one parameter, a combination of parameters) and the nature of the parameters change. These outputs are combined with the outputs of the neural network ANN, determined by the level of risk of an accident on the basis of check-lists, and are appointed by the inputs of the multilayer network to make decisions on the rapid assessment of the crane state on the basis of incoming information in real time, as well as forecasting work crane based on arbitrary input information streams.

The second considered approach to the analysis of telemetry data is preliminary processing of the time series within the selected window by the selected telemetry parameter $p\left(t_{k}\right), k=1,2, . ., 20(\Delta T=20)$

For this, on the basis of the least squares method, an approximation dependence of the quantity $p(t)$ in the form of a square polynomial is calculated

$$
p(t) \approx f(t)=a+b \cdot t+c \cdot t^{2}
$$

In this case, the value $r_{1}=a+10 b+400 c / 3$ determines the average value of the recorded parameter over the interval,

$r_{2}=b+20 c$ is the average value of the speed on the interval,

$r_{3}=2 \mathrm{c}$ - average acceleration value,

$r_{4}=\sum_{k=1}^{20}\left(p\left(t_{k}\right)-f\left(t_{k}\right)\right)^{2}$ - the root-mean-square error of approximation, proportional to the influence on the recorded parameter of random processes of a natural nature or control mechanisms.

As a result, the inputs of RNN in this case are not the temporal values of the parameter within the selected window, but the values, which reduces the number of neurons in the input layer, reduces the network learning time and the network operation error.

The general scheme of the risk assessment process for the safe operation of the facility is shown in Figure 3. 


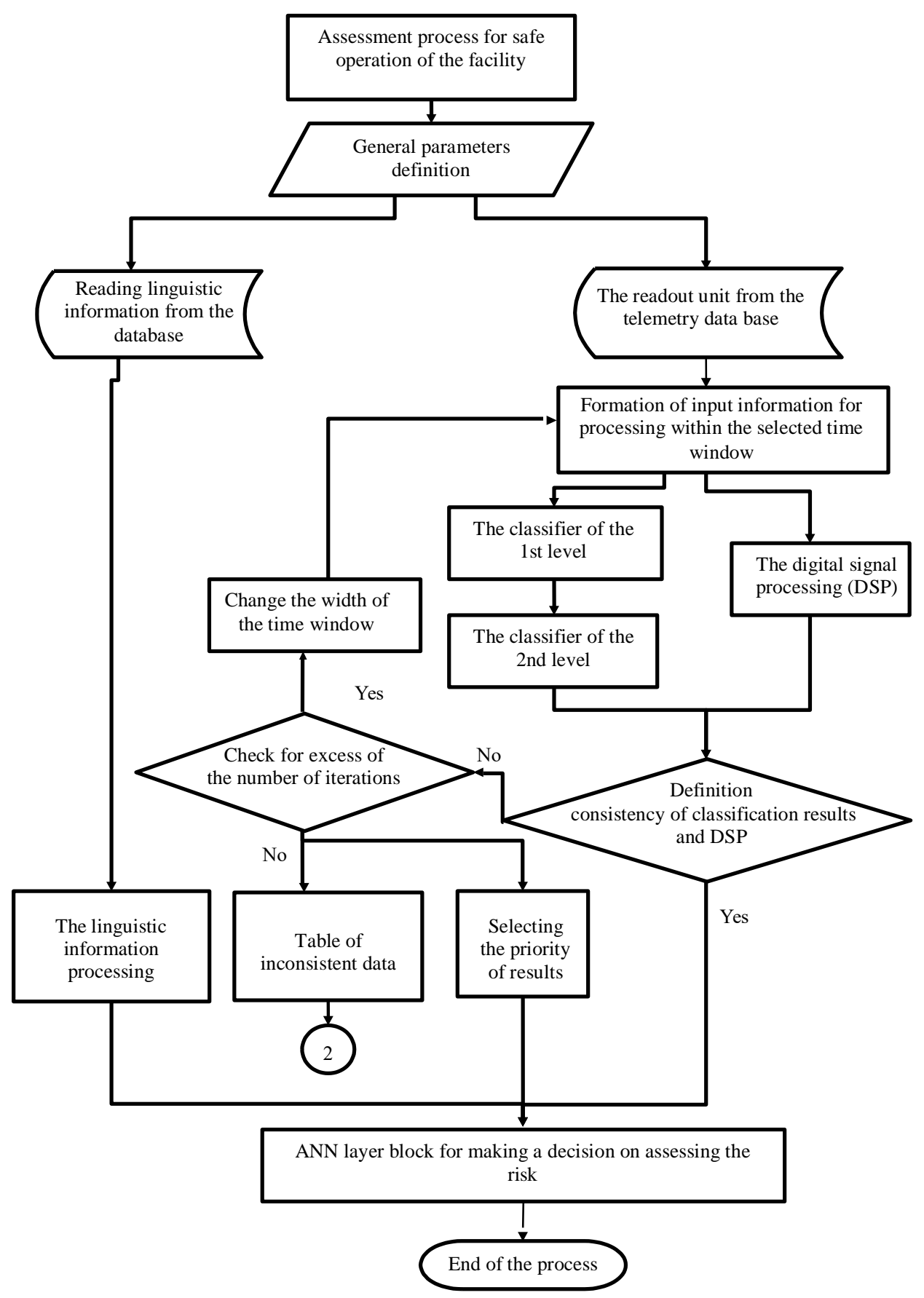

Fig. 3. General scheme of the risk assessment process for safe operation of the facility.

The description of the purpose of the circuit blocks is as follows:

1. General parameters definition block. The beginning and the length of the telemetry time series sample of the selected crane are determined. 
2. The readout unit from the telemetry data base reads it from the table and transfers it to Python data arrays.

3. The block for the formation of input information for processing within the selected time window implements the preparation of information as inputs to neural networks classifiers of the 1 st and 2 nd levels.

4. The classifier of the 1 st level classifies the time series according to the types of the physical process implemented on the crane: lifting of the load, movement of the carriage, movement along the rails, turning, entering the zone of coordinate protection, approaching the zone of limiting the carrying capacity.

5. The classifier of the 2 nd level classifies information for time series by the nature of the change into the following classes: no change, uniform change, accelerated change, random change.

6. The digital signal processing block calculates the numerical characteristics $r_{i}, i=$ $1,2,3,4$ for their comparison in the block Determination of the consistency of the classification and DSP results with the recognized signal membership classes

7. The Check for excess of the number of iterations and Change the width of the time window blocks regulate the matching process by decreasing the length of the time series with a limit on the number of iterations of changing the given length.

8. In case of unsatisfactory work of the classifiers, telemetry data is included in the table of inconsistent data for network retraining (mark 2)

9. The block for reading linguistic information from the database on checklists implements reading information from the table and fuzzifies the corresponding values of linguistic variables.

10. The linguistic information processing unit prepares information as inputs to ANN.

The ANN layer block for making a decision on assessing the risk of safe operation of an object at this stage of the project implementation, together with block 10, is a single fourlayer ANN, the study of which is described above

\section{Conclusions}

Thus, based on the analysis of the tasks of processing the input operational and statistical information, it can be concluded that the use of neural network methods has advantages in relation to the use of algorithmic methods for detecting dangerous conditions during the operation of cranes according to the following characteristics:

- Within the framework of the implemented information system, telemetry data on the operation of lifting crane devices are institutional in nature, accumulates over time and reduces the degree of uncertainty in solving the risk problem. At the same time, neural networks have the property of learning and retraining, which allows them to be successfully used in the conditions of accumulating statistical data;

- artificial neural networks have the property of identifying unknown patterns that lie in the structure of big data. This, in the context of the analysis of significant information flows, will allow not only to adequately describe the operational reliability of the crane, but also to predict the behavior of the object in conditions of a lack of telemetric information on emergency situations;

- the neural network approach allows one to use the assessment of heterogeneous information entering the system being developed from a unified position: having a fuzzy nature and telemetric time data;

- in the operating mode after training the neural network, the computing mechanism is the fastest, which for the development of a system operating in real time is a necessary 
element for maintaining its stability and scalability;

- neural networks are resistant to weak signals and noise, which is one of the important conditions for the reliability of information processing processes for the analysis of telemetry data;

- in the project the neural networks do not completely replace algorithmic methods while are supplemented with digital signal processing algorithms and statistical methods, which increases the reliability of the system being developed.

\section{Acknowledgments}

The work is performed as part of the project "RePROTECT" (IT Software development to assist in decision making process of preventing accidents at the industrial sites of increased risks with the use of a risk-based approach). The project is being done with the financial support of Foundation for Assistance to Small Innovative Enterprises in Science and Technology (FASIE) under Agreement \#78ГРЦТС10-D5 / 56118 / 18.12.2019 - 17.01.2021.

\section{References}

1. Korotkiy A A, Kinzhibalov A A, Panfilov A V and Kurilkin A D 2016 Bezopasnost truda $v$ promyshlennosti 2

2. Panfilov A V, Bakhteyev O A, Deriyshev V V and Korotkiy A A 2020 Safety of Technogenic and Natural Systems 2

3. Bui X-N, Nguyen H, Choi Y, Nguyen-Thoi T, Zhou J and Dou J 2020 Scientific Reports 10(1)

4. Wu D, Yuan Y, Huang J and Tan Y 2020 IEEE Transactions on Fuzzy Syst. 28(5)

5. Wang L-X and Mendel J M 1992 IEEE Int. Conf. Fuzzy Syst. (San Diego CA USA) p 1409

6. Seon Park H, Hwan An J, Jun Park Y and Kwan Oh B 2020 Expert Systems with Applications 158113634

7. Abduljabbar R, Dia H, Liyanage S and Bagloee S A 2019 Applications of artificial intelligence in transport: an overview Sustainability 11(1) 189

8. Cao M T, Tran Q V, Nguyen N M and Chang K T 2020 Advanced Eng. Informatics 46 101182

9. Panchalingam R and Chan K C 2019 Intelligent Buildings International

10. Wang Yu R, Zou Y and Wang Z 2020 Transportation Research Part C: Emerging Technologies 119102740

11. Beskopylny A, Lyapin A, Anysz H, Meskhi B, Veremeenko A and Mozgovoy A 2020 Materials 132445

12. Poh C Q X, Ubeynarayana C U and Goh Y M 2018 Automation in Construction 93 